\title{
Rol de la suplementación de selenio en el tratamiento del hipotiroidismo
}

\author{
Angélica María González Clavijo(D) 1, Juan David Muñoz Loaiza(iD2, \\ Alejandro Márquez Fernández (D)2, Laura Marcela Chávez Rodríguezidz \\ Laura Alejandra Campos GonzáleziD2, David Felipe Alfonso Cedeño(iD2, \\ Luis Felipe Rojas Serna \\ ${ }^{1}$ Departamento de Ciencias Fisiológicas, Facultad de Medicina, Universidad Nacional \\ de Colombia, Bogotá, Colombia \\ ${ }^{2}$ Facultad de Medicina, Universidad Nacional de Colombia, Bogotá, Colombia
}

Cómo citar: González-Clavijo AM, Muñoz-Loaiza JD, Márquez-Fernández A, Chávez-Rodríguez LM, Campos-González LA, Alfonso-Cedeño DF, et al. Rol de la suplementación de selenio en el tratamiento del hipotiroidismo. Rev. Colomb. Endocrinol. Diabet. Metab. 2021;8(2):e603. https://doi.org/10.53853/encr.8.2.603

Recibido: 22/Sept/2020

Aceptado: $11 / 0 c t / 2021$

Publicado: 15/Oct/2021

\section{Resumen}

Introducción: el hipotiroidismo es una endocrinopatía caracterizada por la acción o la producción deficiente de las hormonas tiroideas en el organismo. Una de las etiologías menos frecuentes es la disfunción en las desyodasas, la cual puede sospecharse cuando el perfil tiroideo evidencia hormona tiroestimulante (TSH) elevada, tiroxina (T4) libre alta con triyodotironina (T3) total o libre baja.

Objetivo: dar a conocer un caso de hipotiroidismo tratado exitosamente con selenio y levotiroxina, así como describir el papel del selenio en la enfermedad tiroidea.

Presentación del caso: hombre de 22 años con alteraciones en el perfil tiroideo caracterizado por TSH y T4 libre altas y T3 en el límite inferior, sin hallazgo de adenomas de hipófisis, mutaciones en el gen receptor de hormona tiroidea (THRB) o alteraciones en las globulinas transportadoras de tiroxina, que se trató exitosamente con suplencia de hormona tiroidea y selenio.

Discusión y conclusión: frente a concentraciones anómalas en los niveles de TSH, T4 y T3, no fácilmente explicables por otras causas, las alteraciones en la síntesis o función de las desyodasas deben considerarse. El tratamiento con selenio y levotiroxina podría ayudar en el manejo de esta patología.

Palabras clave: selenio, selenoproteína, hipotiroidismo, triyodotironina, tiroxina.

Correspondencia: Angélica María González-Clavijo, Carrera 30 No. 45-03, edificio 471, piso: 4, oficina 406, Departamento de Fisiología, Facultad de Medicina, Universidad Nacional de Colombia. Correo electrónico: angelik_md@yahoo.com 


\section{Role of selenium substitution in the treatment of hypothyroidism}

\section{Abstract}

Introduction: Hypothyroidism is an endocrinopathy characterized by deficient action or production of thyroid hormones in the body. One of the less frequent etiologies is dysfunction of the deiodinase enzymes, which can be suspected when the thyroid profile shows elevated thyroid-stimulating hormone (TSH), high free thyroxine (T4) and low total or free triiodothyronine (T3).

Purpose: To discuss a case of hypothyroidism successfully treated with selenium and levothyroxine and to describe the role of selenium in thyroid disease.

Case presentation: 22-year-old man with abnormal thyroid profile characterized by elevated $\mathrm{TSH}$, high free T4, and T3 in the lower limit, without finding of pituitary adenomas, mutations in the thyroid hormone receptor gene (THRB) or alterations in the Thyroxine-binding globulin, who was successfully treated with thyroid hormone and selenium supplementation.

Conclusion: when abnormal concentrations in TSH, T4 and T3 levels are not easily explained by other causes, disorders of the synthesis or function of deiodinases should be considered. Treatment with selenium and levothyroxine could help in the management of this pathology.

Key-words: Hypothyroidism, Selenium, Thyroxine, Triiodothyronine, Endocrine System Diseases.
Destacados

- El desconocimiento de etiologías de hipotiroidismo menos frecuentes como la disfunción en desyodasas, conlleva al retraso en el diagnóstico y el tratamiento.

- Debido a la naturaleza de las desyodasas como selenoproteínas, la suplementación con el selenio podría mejorar la función biológica de estas enzimas.

- El rol del selenio en la patología tiroidea se ha estudiado de manera amplia para la enfermedad autoinmune; se necesitan más estudios para la evaluación de la utilidad la patología no autoinmune.

\section{Introducción}

Las hormonas secretadas por la glándula tiroides $(T 4, T 3)$ son importantes para diversos procesos en el cuerpo, como la regulación del metabolismo de lípidos, el ritmo cardiaco, la temperatura, el correcto funcionamiento cognitivo, la tasa metabólica basal, entre otros (1). La falta de esta hormona (bien sea por problemas centrales o periféricos) conlleva al hipotiroidismo, el cual se puede manifestar con fatiga, letargo, aumento de peso, estreñimiento, cognición alterada e infertilidad, entre muchos otros síntomas $(2,3)$.

Una de las causas de hipotiroidismo menos documentadas es la deficiencia de desyodasas, selenoproteínas que participan en el catabolismo de las hormonas tiroideas (4). Específicamente, la desyodasa tipo 2 (D2) es la principal responsable por la conversión de T4 a T3, la forma activa de estas hormonas (5). La disfunción en esta proteína ocasionaría altos niveles de T4 y bajos niveles de T3, desencadenando no solo el déficit de la actividad hormonal tiroidea, sino una elevación de los niveles de TSH debido a la pérdida del retrocontrol negativo (6).

El desconocimiento de esta forma de hipotiroidismo podría llevar a los médicos tratantes a una demora en el diagnóstico y el tratamiento oportuno. A continuación, se realiza un caso clínico de difícil diagnóstico de un paciente joven con presunta disfunción en desyodasas, tratado con levotiroxina (LT4) y selenio. 


\section{Presentación del caso clínico}

Paciente de 22 años consulta por diaforesis de larga data de predominio en manos y pies, que aumenta con el estrés. Dentro de sus antecedentes familiares destaca hipotiroidismo. Al examen físico el único hallazgo relevante es bocio grado I, según la Organización Mundial de la Salud (OMS). Se le pide perfil tiroideo en el cual se encuentra TSH en 7,79 ng/dl y T4 libre en 1,76 ng/dl (figura 1, fecha 2016/02). Se le deja seguimiento sin tratamiento farmacológico.

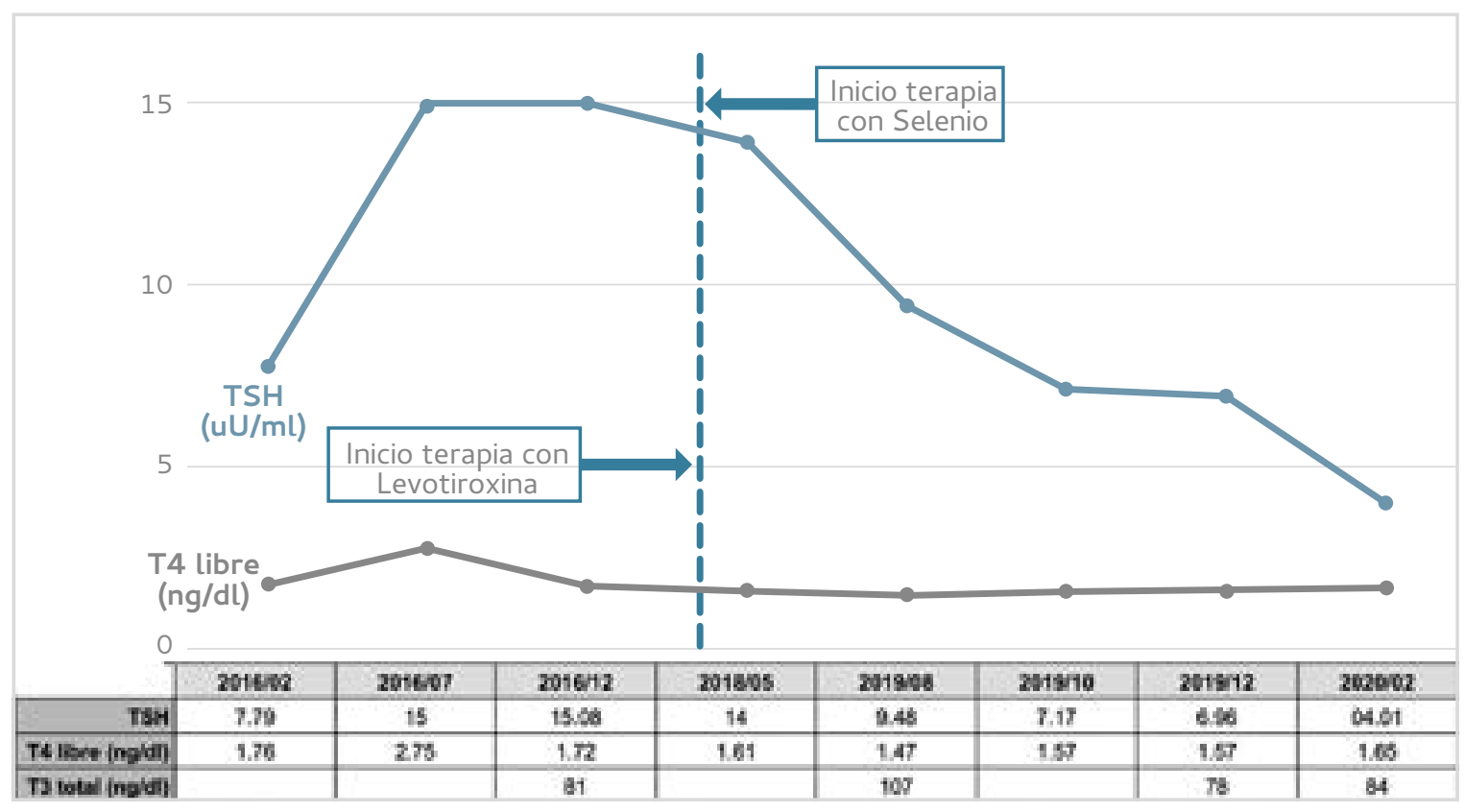

Figura 1. Evolución del perfil tiroideo

Fuente: elaboración propia.

A los cinco meses, se repite el perfil tiroideo encontrándose TSH y T4 libre que superan los valores previos (figura 1 , fecha 2016/07). En los paraclínicos se encuentra perfil lipídico, función hepática, creatinina, prolactina y testosterona en niveles normales, pero llaman la atención los niveles de TSH, T4 totaly $\mathrm{T} 4$ libre altos para el punto de corte del laboratorio, mientras que T3 total y T3 libres son normales (figura 1, fecha 2016/12). La ecografía mostró un aumento en el volumen tiroideo, una disminución de la ecogenicidad del parénquima y múltiples lesiones microquísticas. Los niveles de anticuerpos anti-TPO se encuentran normales.

Las alteraciones en el perfil tiroideo sugieren hipertiroidismo secundario a adenoma productor de TSH, por lo que se solicita una resonancia magnética de hipófisis, la cual resulta normal. Debido a que no se encontraron hallazgos significativos a nivel central y para evaluar 
una posible resistencia a hormonas tiroideas derivado de problemas en el receptor de $\mathrm{T3}$, se ordena un estudio genético del gen receptor de hormona tiroidea (THRB), el cual no mostró deleciones o duplicaciones de gran tamaño. En adición, la globulina de unión a tiroxina se encuentra dentro del rango de normalidad.

Meses después, el paciente vuelve a consultar refiriendo síntomas de adinamia y somnolencia. Su examen físico se encuentra sin hallazgos patológicos además del bocio ya descrito, por lo que se decide pedir un nuevo perfil tiroideo. Los resultados nuevamente presentan el patrón de TSH alto, T4 en el límite superior y T3 en el límite inferior, debido a estos hallazgos y por la historia clínica previa, se sospecha de una alteración en las desyodasas que limita la conversión de T4 a T3. Se indica manejo con hormona tiroidea y selenio, lográndose el estado eutiroideo con una dosis de 75 ug de levotiroxina y 200 ug de selenio (figura 1 ). Después de un año de seguimiento el paciente sigue eutiroideo y asintomático.

\section{Discusión}

El hipotiroidismo se encuentra dentro de las endocrinopatías más prevalentes, siendo el género femenino el más afectado (8). A pesar de que la causa más común de hipotiroidismo se relaciona con problemas autoinmunes (Hashimoto) en países con suficiente ingesta de yodo, y a la carencia de este en países donde no la hay (9), es importante tener en cuenta otras etiologías poco frecuentes y menos conocidas, como por ejemplo la alteración en las desyodasas.
Las desyodasas son enzimas importantes en el catabolismo de las hormonas tiroideas, perteneciendo al selecto grupo de las selenoproteínas, las cuales se caracterizan por tener al menos un residuo del aminoácido selenocisteína.

Existen tres tipos de desyodasas (D1, D2 y D3) responsables de la regulación periférica de la actividad hormonal tiroidea y estas actúan removiendo grupos específicos de yodo de estas hormonas. Se teoriza que la selenocisteína actúa como el aceptor final del yodo en la reacción (10).

Las tres desyodasas difieren en localización tisular y blanco; D2 es una desyodasa de anillo externo, localizada en hipófisis, músculo esquelético, corazón y riñón (11). Si bien D1 y D2 producen T3 activa a partir de T4, la segunda es más efectiva por encontrarse en el retículo endoplasmático y no en la membrana como D1, facilitando la entrada de la molécula por su cercanía al núcleo (12). A pesar de que la glándula tiroides en condiciones normales secreta tanto T4 como T3, el $80 \%$ de T3 es producido por la conversión desde T4 en la periferia.

A nivel del hipotálamo, las células ependimarias localizadas entre el tercer ventrículo y la eminencia media son LAs principales productoras de T3, debido a que poseen D2 (13). Lo mismo sucede en la adenohipófisis con las células estrelladas foliculares, ambas células dotan al encéfalo de T3 que después se une a los receptores nucleares de esta hormona, contribuyendo así a la retroalimentación negativa; por ello, problemas en la desyodasa 2 causarían pérdida del retrocontrol negativo. En adición, la acción de D3 que convierte de T3 a su forma inactiva (T3 reversa) (14) 
podría agravar el problema, disminuyendo aún más los niveles de triyodotironina a nivel central, dando un perfil tiroideo como el del paciente: niveles de TSH y T4 aumentadas, mientras que T3 está disminuido o en el límite inferior (15). Debido a que T3 es necesario para realizar las acciones genómicas de las hormonas tiroideas, la disfunción de D2 tendría como consecuencias síntomas del hipotiroidismo.

Con el hallazgo de hace 50 años de que, en los humanos, las desyodasas producen la mayor parte de la T3 circulante, se reforzó la idea de que la monoterapia con levotiroxina mantendría la reserva de T4 y las desyodasas proporcionarían una regulación fisiológica de la disponibilidad de T3 para los tejidos $(16,17)$. Sin embargo algunos pacientes, pese a tener valores de TSH en rangos, no logran normalizar por completo los valores de T3 debido a una conversión insuficiente de T4 a T3, lo que explicaría la persistencia de síntomas a pesar del tratamiento. Estos casos han llevado a explorar alternativas complementarias a la monoterapia con levotiroxina, como el selenio o la liotironina sódica.

La liotironina sódica es un análogo de T3 que si bien no está en el PBS (plan de beneficios de salud) puede encontrarse en presentaciones de tabletas de 25 ug o como solución inyectable de $10 \mathrm{mg} /$ $\mathrm{ml}$. Su uso como terapia combinada con levotiroxina ha sido evaluado en múltiples estudios y con diferentes proporciones de T3:T4. Algunos informes respaldan su uso pero otros no concluyen que ayuden en el tratamiento $(18,19,20)$.

En los pacientes cuyo hipotiroidismo es de origen primario o secundario, los obstáculos que se oponen al éxito del tratamiento podrían tener explicación en la biología enzimática de D2, la cual si bien tiene alta afinidad por T4 y realiza la conversión a T3 en hipotálamo y periferia, tiene una vida media diferente en ambas regiones (17).

A nivel central, la T4 debe convertirse en T3, principalmente para suprimir la secreción de TSH y a nivel periférico para lograr un pool necesario de la hormona biológicamente activa que actúe en los tejidos, y cada vez que se convierte T4 en T3, D2 se inactiva y degrada.

A nivel periférico la tasa de conversión de T4 a T3 disminuye progresivamente con la administración de la hormona tiroidea debido a la ubiquitinación de D2, de modo que la producción periférica de T3 de los pacientes hipotiroideos tratados con levotiroxina disminuye paulatinamente y se autorregula; no así con la D2 hipotalámica que es menos susceptible a la ubiquitinación y posterior degradación proteosémica.

Como resultado, la dosis de levotiroxina requerida para normalizar las concentraciones séricas de TSH es más baja que la dosis que normaliza los niveles séricos de T3 (17).

En investigaciones relacionadas con la D2, se ha descrito el polimorfismo Ala92-Dio2 como causante del hipotiroidismo, pues una mutación impide la ubiquitinación de la desyodasa, provocando estrés reticular, acumulación en trans-Golgi y una muy baja tasa de conversión a T3. Estudios con ratones demostraron que la administración de la chaperona fenilbutirato de sodio (4PBA) corregiría la disminución de T3 al promover la correcta eliminación de la enzima y así llevar a niveles eutiroideos 
globales, contrarios a los efectos locales que promueve la levotiroxina (21). Al tener en cuenta los recientes hallazgos, sería de gran ayuda diagnóstica que los pacientes asociados a hipotiroidismo por desyodasas se sometan a una tipificación del gen de la D2 (DIO2), ya que esto permitiría la identificación precisa de la etiología, reduciendo además el tiempo de diagnóstico.

El rol del selenio en patologías asociadas a la tiroides se ha venido estudiando desde hace décadas (22), sin embargo, este oligoelemento aún no es usado como un tratamiento de rutina, en gran parte por falta de evidencia. Dentro de los mecanismos de acción que podría tener el selenio en dichas patologías destaca su rol en la regulación del sistema inmune (23). En algunos estudios realizados con ratones deficientes en selenio, el conteo de células $T$ funcionales disminuye, así como su capacidad de suprimir los radicales libres; las células $T$ reguladoras no pueden proliferar en un medio con estrés oxidativo, por tanto, no pueden limitar la producción de interleucinas como IL-2, perdiendo el control sobre los linfocitos $T$ autorreactivos y sobre los linfocitos $B$ que producen autoanticuerpos que puedan causar una patología a nivel de la tiroides (24). Estos mecanismos propuestos cobran sentido porque, además de las desyodasas, dentro de las selenoproteínas también se encuentran moléculas que protegen del estrés oxidativo como la enzima glutatión peroxidasa.

En varios estudios realizados en pacientes con enfermedad de Graves se ha encontrado que estos logran el eutiroidismo más rápidamente en una terapia de medicamentos antitiroideos combinada con selenio que los pacientes que solo reciben monoterapia (25). En otro ensayo se le administró selenio a pacientes eutiroideos con orbitopatía de Graves leve, observándose una reducción de la enfermedad (26). De la misma manera, se han realizado investigaciones para evaluar el potencial uso del selenio en pacientes con tiroiditis de Hashimoto, dando como resultado una disminución en los niveles de anticuerpos anti-TPO, sin un cambio en los niveles de TSH o las hormonas tiroideas $(27,28)$. Estos hallazgos comienzan a vislumbrar el papel importante que podría tener el selenio en el tratamiento de la patología tiroidea.

En el presente caso, al sospecharse un problema con la desyodasa D2, la administración de selenio mejoraría la biodisponibilidad hormonal al ser el precursor del aminoácido selenocisteína, normalizando así el perfil tiroideo como se cree que ocurrió en el paciente. Debido a que no hay evidencia suficiente que respalde este desenlace, se abre la puerta a estudios que permitan enjuiciar la participación del selenio en el tratamiento de la patología tiroidea no autoinmune.

Dentro de las limitaciones en este caso, reportamos que debido a las condiciones que se tienen en el medio, a este paciente no se le pudieron medir los niveles de selenio antes y después del tratamiento, por lo que no se puede corroborar si realmente el medicamento administrado tuvo un impacto en los niveles séricos. Sumado a esto, tampoco fue posible realizar un estudio genético para los genes que codifican las desyodasas, para así verificar una posible 
mutación en estos. Por tales motivos, el enfoque diagnóstico se hizo a través de la prueba terapéutica, la mejoría de la sintomatología y otros paraclínicos.

En Colombia, el uso del selenio está limitado por su ausencia como un medicamento en farmacias, solo está disponible en algunas tiendas naturistas las cuales no están tan ampliamente distribuidas a lo largo del territorio y no ofrecen los mismos productos. Además, no hace parte del Plan de Beneficios en Salud (PBS), lo cual es una limitante económica para adquirir el oligoelemento.

\section{Conclusiones}

La falta de información sobre el diagnóstico y el tratamiento de las causas menos comunes relacionadas al hipotiroidismo, lleva a que los pacientes con estas enfermedades tengan experiencias con tratamientos largos y múltiples diagnósticos fallidos, antes del descubrimiento de etiologías como el hipotiroidismo por disfunción en las desyodasas. Se presume que el eutiroidismo se podría conseguir a través de tratamiento empírico con levotiroxina combinada con selenio.

El reporte de este caso y la discusión de sus aspectos diagnósticos y biológicos permitirá a la comunidad académica considerar una causa poco frecuente de hipotiroidismo, pero que podría tratarse exitosamente con la terapia usual de levotiroxina y la suplementación con selenio.

\section{Consentimiento informado}

El paciente autorizó la publicación del caso clínico.

\section{Declaración de fuentes de financiación}

Esta investigación no recibió ningún soporte financiero.

\section{Conflicto de intereses}

Los autores declaran que no tuvieron ningún conflicto de interés asociado al desarrollo de la investigación.

\section{Referencias}

[1] Pirahanchi Y, Tariq MA, Jialal I. Physiology, Thyroid. 2020;1-7. En: StatPearls [Internet]. Treasure Island (FL): StatPearls Publishing; 2021. https://www.ncbi.nlm.nih. gov/books/NBK519566/

[2] Chaker L, Bianco AC, Jonklaas J, Peeters RP. Hypothyroidism. Lancet. 2017;390(10101):155062. https://doi.org/10.1016/S01406736(17)30703-1

[3] Persani L, Cangiano B, Bonomi $M$. The diagnosis and management of central hypothyroidism in 2018. Endocr Connect. 2020;8(2):R4454. https://doi.org/10.1530/EC-180515

http://revistaendocrino.org/index.php/rcedm 
[4] Gomes-Lima C, Wartofsky L, Burman K. Can Reverse T3 Assay Be Employed to Guide T4 vs. T4/ T3 Therapy in Hypothyroidism? Front Endocrinol. $2019 ; 10$ (diciembre): 1 - 5 . https://doi.org/10.3389/ fendo.2019.00856

[5] Park E, Jung J, Araki O, Tsunekawa K, Park SY, Kim J, etal. Concurrent TSHR mutations and DIO2 T92A polymorphism result in abnormal thyroid hormone metabolism. Sci Rep. 2018;8(1):1-19. https:// doi.org/10.1038/s41598-01828480-0

[6] Hadlow NC, Rothacker KM, Wardrop R, Brown SJ, Lim EM, Walsh JP. The relationship between $\mathrm{TSH}$ and free T4in a large population is complex and nonlinear and differs by age and sex. J Clin Endocrinol Metab. 2013;98(7):2936-43. https://doi.org/10.1210/jc.20124223

[7] Winther $\mathrm{KH}$, Watt $\mathrm{T}$, Bjørner JB, Cramon P, Feldt-Rasmussen $U$, Gluud C, et al. The chronic autoimmune thyroiditis quality of life selenium trial (CATALYST): Study protocolfor a randomized controlled trial. Trials. 2014;15(1):1-20. https://doi.org/10.1186/1745-621515-115

[8] Bernardi LA, Cohen RN, Stephenson MD. Impact of subclinical hypothyroidism in women with recurrent early pregnancy loss. Fertil Steril [Internet]. 2013;100(5):132631.e1. https://doi.org/10.1016/j. fertnstert.2013.07.1975
[9] Almandoz J, Gharib $H$. Hypothyroidism: Etiology, Diagnosis, and Management. Med Clin N Am. 2012;96(2):20321 . https://doi.org/10.1016/j. mcna.2012.01.005

[10] Zhang Y, Roh YJ, Han S, Park I, Lee HM, Ok YS, et al. Role of selenoproteins in redox regulation of signaling and the antioxidant system: a review. Antioxidants (Basel). 2020my.;9(5):383.https:// doi.org/10.3390/antiox9050383

[11] Bernal J. Síndromes de resistencia a las hormonas tiroideas. Endocrinol y Nutr. 2011;58(4):18596. https://doi.org/10.1016/j. endonu.2011.02.001

[12] Bianco A, da Conceição R. The deiodinase trio and thyroid hormone signaling. Method Mol Biol. 2018;67-83. https://doi. org/10.1007/978-1-4939-7902-8_8

[13] Fekete C, Mihály E, Herscovici $S$, Salas J, Tu $H$, Larsen PR, et al. DARPP-32 and CREB are present in type 2 iodothyronine deiodinase-producing tanycytes: implications for the regulation of type 2 deiodinase activity. Brain Res. 2000;862(1-2):154-61. https://doi.org/10.1016/S00068993(00)02105-3

[14] Huang SA. Physiology and pathophysiology of type 3 deiodinase in humans. Thyroid. 2005;15(8):875-81. https://doi. org/10.1089/thy.2005.15.875

[15] Dumitrescu AM, Liao XH, Abdullah MSY, Lado-Abeal J, Majed FA, Moeller LC, et al. Mutations in 
SECISBP2 result in abnormal thyroid hormone metabolism. Nat Genet. $\quad$ 2005;37(11):1247-52. https://doi.org/10.1038/ng1654

[16] McAninch EA, Bianco AC. New insights into the variable effectiveness of levothyroxine monotherapy for hypothyroidism. Lancet Diabetes Endocrinol. 2015;3(10):756-8. https://doi. org/10.1016/S2213-8587(15)00325-3

[17] Gereben B, McAninch EA, Ribeiro MO, Bianco AC. Scope and limitations of iodothyronine deiodinases in hypothyroidism. Nat Rev Endocrinol. 2015;11:642-52.

https://doi.org/10.1038/ nrendo.2015.155

[18] Shin D, Kim K, An J, Lee E. Different TSH suppressive effects of liothyronine combination according to Thr92Ala type 2 deiodinase polymorphism. Endocrine Abstracts. 2015;37. https://doi.org/10.1530/ endoabs.37.0C6.3

[19] Jo S, Fonseca T, Bocco B, Fernandes G, McAninch E, Bolin A, et al. Type 2 deiodinase polymorphism causes ER stress and hypothyroidism in the brain. J Clin Invest. 2018;129(1):230-45. https://doi. org/10.1172/JCl123176

[20] Fischman A, Domínguez J. Combined therapy with levothyroxine and liothyronine for hypothyroidism. Medwave. 2018;18(08):e7376-e7376. https://doi.org/10.5867/ medwave.2018.08.7375

[21] Jo S, Fonseca TL, Bocco BMLC, Fernandes GW, McAninch EA,
Bolin AP, et al. Type 2 deiodinase polymorphism causes ER stress and hypothyroidism in the brain. J Clin Invest. 2019 Jan 2;129(1). https:// doi.org/10.1172/JCI123176

[22] Sánchez A. Selenio y tiroides. Rev tiroides. 2009;18:40-45.

[23] Valea A, Georgescu CE. Selenoproteins in human body: focus on thyroid pathophysiology. Hormones. 2018;17:183-96. https://doi.org/10.1007/s42000018-0033-5

[24] Ventura M, Melo M, Carrilho F. Selenium and Thyroid Disease: From Pathophysiology to Treatment. Int J Endocrinol. 2017;2017:1-9. https://doi. org/10.1155/2017/1297658

[25] Winther K, Rayman M, Bonnema $S$, Hegedüs L. Selenium in thyroid disorders - essential knowledge for clinicians. Nature Rev Endocrinol. 2020;16(3):165-76. https://doi. org/10.1038/s41574-019-0311-6

[26] Vrca VB, Skreb F, Cepelak I, Romic $Z$, Mayer L. Supplementation with antioxidants in the treatment of Graves' disease; the effect on glutathione peroxidase activity and concentration of selenium. Clin Chim Acta. 2004;341(1-2):55-63.

\section{https://doi.org/10.1016/j. cccn.2003.10.028}

[27] Marcocci C, Kahaly GJ, Krassas GE, Bartalena L, Prummel M, Stahl M, et al. Selenium and the course of mild Graves' orbitopathy. N Engl J Med. 2011;364:1920-31. https://doi. org/10.1056/NEJMoa1012985 
[28] Gartner R, Gasnier BC, Dietrich JW, Krebs B, Angstwurm MW. Selenium supplementation in patients with autoimmune thyroiditis decreases thyroid peroxidase antibodies concentrations. J Clin Endocrinol Metab. 2002;87:1687-91. https:// doi.org/10.1210/jcem.87.4.8421 